12

\title{
Теория и расчет электростатических электронных зеркал с учетом релятивистских эффектов
}

\author{
(C) С.Б. Бимурзаев, Е.М. Якушев
}

Алматинский университет энергетики и связи им. Г. Даукеева, 050013 Алматы, Казахстан

e-mail: bimurzaev@mail.ru

Поступило в Редакцию 12 октября 2020 г.

В окончательной редакции 30 ноября 2020 г.

Принято к публикации 1 декабря 2020 г.

С помощью метода центральной частицы получены уравнения траектории заряженных частиц с точностью до величин третьего порядка малости включительно в осесимметричном электростатическом зеркале с учетом релятивистских эффектов. Определены условия пространственной фокусировки и коэффициенты пространственных аберраций в гауссовой плоскости изображения зеркала при учете релятивистских эффектов. Путем численных расчетов определены условия одновременного устранения сферической и осевой хроматической аберраций при учете релятивистских эффектов в осесимметричном электростатическом зеркале, когда предметная плоскость зеркала совмещена с его фокальной плоскостью. Показано, что учет высоких скоростей частиц приводит как к смещению положения гауссовой плоскости изображения, так и изменению качества фокусировки.

Ключевые слова: электронный микроскоп, электростатическое зеркало, сферическая аберрация, осевая хроматическая аберрация, релятивистский эффект.

DOI: $10.21883 /$ JTF.2021.05.50701.290-20

\section{Введение}

В настоящее время электронные зеркала стали незаменимыми структурными элементами современного научного и технологического приборостроения, определяющие качество фокусировки таких приборов, как массспектрометры и электронные микроскопы. Реальное конструирование подобных приборов требует предельно точного расчета электронно-оптического тракта, в том числе учета релятивистских поправок, особенно в области электронной микроскопии. Как известно, главными факторами, ограничивающими разрешающую способность электронного микроскопа, являются сферическая и осевая хроматическая аберрации электронной линзы, выполняющей роль его объектива $[1,2]$. К настоящему времени достигнуты значительные успехи в разработке корректоров аберраций, позволяющих одновременное устранение сферической и хроматической аберраций объективной линзы электронного микроскопа как на основе мультипольных электрических и магнитных полей, так и на основе электростатического зеркала [39]. Однако в известных работах не учитывается влияние релятивистских эффектов на качество фокусировки, что особенно необходимо для высоковольтной (с ускоряющим напряжением $100 \mathrm{keV}$ и выше) электронной микроскопии. Настоящая работа посвящена созданию теории пространственных аберраций и расчету электростатического осесимметричного зеркала при учете влияния релятивистских эффектов на его фокусирующие свойства.

\section{1. Уравнения траекторий}

\section{1. Уравнения траекторий в подвижной системе координат}

Введем цилиндрическую систему координат $r, \psi, z$, ось $z$ которой совместим с главной оптической осью зеркала. Электростатическое поле зеркала представим скалярным потенциалом $\varphi=\varphi(r, z)$, нормированным так, что в месте поворота частицы (где кинетическая энергия некоторой выбранной частицы равна нулю) и $\varphi \equiv \varphi_{0}$ в свободном от поля пространстве. Рассмотрим поток однородных частиц с зарядом $e$ и массой покоя $m_{0}$, движущихся в меридиональных плоскостях $\psi=$ const в окрестности главной оптической оси зеркала. При этих условиях вариационная функция Лагранжа $L$ может быть представлена в виде

$$
L=\sqrt{1-\frac{1}{c^{2}}\left(\dot{r}^{2}+\dot{z}^{2}\right)}-\gamma^{2} \frac{\varphi}{\varphi_{0}} .
$$

Здесь точками, как обычно, обозначено дифференцирование переменных по времени $t$. Безразмерная величина $\gamma^{2}=-e \varphi_{0} / m_{0} c^{2}$, равная кинетической энергии, отнесенной к энергии покоя частиц, представляет собой количественную характеристику релятивистских эффектов поступающих в поле зеркала частиц. При этом уравнения движения частицы принимают вид

$$
\begin{gathered}
\frac{d}{d t}\left(\frac{\dot{r}}{c \sqrt{c^{2}-\dot{r}^{2}-\dot{z}^{2}}}\right)=\frac{\gamma^{2}}{\varphi_{0}} \frac{\partial \varphi}{\partial r}, \\
\frac{c}{\sqrt{c^{2}-\dot{r}^{2}-\dot{z}^{2}}}=1+\gamma^{2}\left(\frac{\varphi}{\varphi_{0}}+\varepsilon\right) .
\end{gathered}
$$


Равенство (3) представляет собой закон сохранения полной энергии частиц. Обычно при исследовании аберраций используют этот закон для исключения времени из уравнений движения и введения в качестве независимой переменной координаты оптической оси $z$. С этим преобразованием связаны определенные математические трудности, вытекающие из того обстоятельства, что в полученных уравнениях появляются структуры типа $\sqrt{\varphi / \varphi_{0}+\varepsilon}$ и $\sqrt{1+r^{\prime 2}}$ (штрихи обозначают дифференцирование по $z$ ). Наличие этих структур при линеаризации уравнений вынуждает принять, наряду с требованием малости $r$, следующие условия параксиального приближения: $\varepsilon \varphi_{0} / \varphi \ll 1$ и $r^{\prime} \ll 1$, которые с очевидностью не могут быть удовлетворены в окрестности точки поворота электронных траекторий в электронном зеркале $\xi=z_{u}$, где $\varphi_{z \rightarrow z_{u}} \rightarrow 0$ и $r_{z \rightarrow z_{u}}^{\prime} \rightarrow \infty$. Поэтому в наших дальнейших исследованиях будем следовать методу „центральной частицы“ $[10,11]$, позволяющему преодолеть указанные трудности.

Сначала с учетом (3) приведем систему уравнений (2)-(3) к виду:

$$
\begin{gathered}
\frac{d}{d t}\left\{\frac{\dot{r}}{c^{2}}\left[1+\gamma^{2}\left(\frac{\varphi}{\varphi_{0}}+\varepsilon\right)\right]\right\}=\frac{\gamma^{2}}{\varphi_{0}} \frac{\partial \varphi}{\partial r}, \\
\frac{1}{c^{2}}\left(\dot{r}^{2}+\dot{z}^{2}\right)=\gamma^{2}\left(\frac{\varphi}{\varphi_{0}}+\varepsilon\right) \\
\times\left[2+\gamma^{2}\left(\frac{\varphi}{\varphi_{0}}+\varepsilon\right)\right]\left[1+\gamma^{2}\left(\frac{\varphi}{\varphi_{0}}+\varepsilon\right)\right]^{-2} .
\end{gathered}
$$

Затем выберем в качестве центральной частицы одну из частиц, движущихся вдоль оси $z$ с $\varepsilon=0$. При этом положение центральной частицы на оси $z$ в текущий момент времени будем обозначать через $\xi$ и свяжем с этой частицей начало подвижной системы координат $r, \psi, \eta$, введя замену переменной

$$
z=\xi+\eta
$$

где $\eta=\eta(\xi)$ - малая величина, определяющая продольное смещение произвольной частицы от центральной. Идея введения подвижной системы координат состоит в том, что исключение времени из уравнений движения в этой системе не приводит к указанным нежелательным последствиям. Кроме того, в подвижной системе координат продольные и поперечные смещения ( $\eta$ и $r$ ) могут рассматриваться как малые величины, следовательно, и относительные скорости частиц $(\dot{\eta}$ и $\dot{r})$ могут оставаться малыми даже при релятивистских скоростях центральной частицы, что несомненно упрощает аберрационный анализ.

Из соотношения (5) найдем скорость центральной частицы

$$
\frac{\dot{\xi}}{c}=\sigma \gamma \frac{\sqrt{\Phi\left(2+\gamma^{2} \Phi\right)}}{1+\gamma^{2} \Phi},
$$

где $\sigma$ - знаковый множитель, характеризующий направление движения частицы вдоль оси $z$, а $\Phi=\Phi(\xi)-$ безразмерная функция аргумента $\zeta$, та же, что и функция
$\Phi(z)=\varphi(o, z) / \varphi_{0}$ аргумента $z$, описывающая осевое распределение потенциала. При этом за пределами поля зеркала $\Phi(\zeta) \equiv 1$, а в особой точке (при $\zeta=z_{u}$ ) имеют место соотношения $\Phi\left(z_{u}\right)=0, \Phi^{\prime}\left(z_{u}\right) \neq 0$, характерные для электронного зеркала.

Используя равенства (6) и (7), введем в уравнения (4) и (5) новую независимую переменную $\xi$ и динамическую переменную $\eta$, исключив из этих уравнений $t$ и $z$. Выполнив соответствующие преобразования, получим

$$
\begin{gathered}
\sqrt{\hat{\Phi} \frac{d}{d \xi}\left(\sqrt{\hat{\Phi}} \frac{d r}{d \xi}\right)} \\
=\frac{1}{2 \varphi_{0}} \frac{\partial \varphi}{\partial r}-\gamma^{2} \sqrt{\hat{\Phi}} \frac{d}{d \xi}\left[\sqrt{\hat{\Phi}}\left(\frac{\varphi}{\varphi_{0}}+\varepsilon\right) \frac{d r}{d \xi}\right] \\
\hat{\Phi}\left[r^{\prime 2}+\left(1+\eta^{\prime}\right)^{2}\right] \\
=\left(\frac{\varphi}{\varphi_{0}}+\varepsilon\right)\left[1+\frac{1}{2} \gamma^{2}\left(\frac{\varphi}{\varphi_{0}}+\varepsilon\right)\right]\left[1+\gamma^{2}\left(\frac{\varphi}{\varphi_{0}}+\varepsilon\right)\right]^{2} .
\end{gathered}
$$

Здесь и далее

$$
\hat{\Phi}=\Phi\left(1+\frac{1}{2} \gamma^{2} \Phi\right)\left(1+\gamma^{2} \Phi\right)^{-2},
$$

а штрихи обозначают дифференцирование по переменной .

Следует отметить, что при выводе уравнений (8) и (9) не вводилось никаких дополнительных ограничений, поэтому эти уравнения справедливы при любых значениях $r, \varepsilon$ и при любых энергиях $\varphi_{0}$ поступающих в систему частиц.

\section{2. Линеаризация уравнений траекторий}

Качество фокусировки определяется аберрациями, понимаемыми как отклонения от параксиального приближения. Одной из основных задач корпускулярной оптики является установление типов аберраций, присущих данной системе, а затем устранение или уменьшение наиболее важных из них. Для определения пространственных хроматических и геометрических аберраций до третьего порядка достаточно в уравнениях траекторий удержать члены не выше третьего малости относительно $r$ и первого порядка - относительно $r \varepsilon$. С этой целью будем искать решения уравнений (8) и (9) для области, близкой к главной оптической оси, и при малых значениях $\varepsilon$.

Из представления о центральной частице следует, что для нее величина $\eta$ равна нулю. Это значит, что существует решение $\eta=\eta(\zeta)$ уравнения (9), величина которого мала при малых значениях $r$ и $\varepsilon$. Мы будем пользоваться именно этим решением.

Используя известное распределение потенциала вблизи оси $z$

$$
\frac{\varphi(r, z)}{\varphi_{0}}=\Phi(z)-\frac{r^{2}}{4} \frac{d^{2} \Phi(z)}{d z^{2}}+\frac{r^{4}}{64} \frac{d^{4} \Phi(z)}{d z^{4}}-\ldots,
$$


а также разложения типа

$$
f(z)=f(\xi+\eta)=f(\xi)+\eta f^{\prime}(\xi)+\ldots,
$$

получим

$$
\begin{gathered}
\sqrt{\Phi\left(1+\frac{1}{2} \gamma^{2} \Phi\right)} \frac{d}{d \xi}\left[\sqrt{\Phi\left(1+\frac{1}{2} \gamma^{2} \Phi\right)} \frac{d r}{d \xi}\right] \\
+\frac{1}{4} \Phi^{\prime \prime} r\left(1+\gamma^{2} \Phi\right)=S+\gamma^{2} S_{\gamma}^{(3)}, \\
2 \tilde{\Phi} \eta^{\prime}-\Phi^{\prime} \eta=\tilde{F},
\end{gathered}
$$

где

$$
\begin{gathered}
S=\frac{1}{32} \Phi^{I V} r^{3}-\frac{1}{4} \Phi^{\prime \prime \prime} r \eta \\
S_{\gamma}^{(3)}=\Phi S-\sqrt{\Phi\left(1+\frac{1}{2} \gamma^{2} \Phi\right)} \\
\times \frac{d}{d \xi}\left[\frac{\delta}{\left(1+\gamma^{2} \Phi\right)} \sqrt{\Phi\left(1+\frac{1}{2} \gamma^{2} \Phi\right)} \frac{d r}{d \xi}\right], \\
\tilde{F}=\varepsilon-\frac{1}{4} \Phi^{\prime \prime} r^{2}-\tilde{\Phi} r^{\prime 2}
\end{gathered}
$$

Здесь и далее

$$
\begin{gathered}
\tilde{\Phi}=\Phi\left(1+\frac{1}{2} \gamma^{2} \Phi\right)\left(1+\gamma^{2} \Phi\right), \\
\delta=\varepsilon+\Phi^{\prime} \eta-\frac{1}{4} \Phi^{\prime \prime} r^{2} .
\end{gathered}
$$

При равенстве нулю правой части уравнения (13) получим уравнение, совпадающее по форме с известным уравнением параксиальных траекторий при высоких скоростях для электростатических линз [12]:

$$
\begin{aligned}
& \sqrt{\Phi\left(1+\frac{1}{2} \gamma^{2} \Phi\right)} \frac{d}{d \xi}\left[\sqrt{\Phi\left(1+\frac{1}{2} \gamma^{2} \Phi\right)} \frac{d r}{d \xi}\right] \\
& +\frac{1}{4}\left(1+\gamma^{2} \Phi\right) \Phi^{\prime \prime} r=0 .
\end{aligned}
$$

Из линейности и однородности этого уравнения относительно $r$ следует, что электростатическое зеркало при релятивистских скоростях электронов способно создавать правильное электронно-оптическое изображение.

Для удобства анализа перепишем (20) следующим образом:

$$
\sqrt{\Phi} \frac{d}{d \xi}\left(\sqrt{\Phi} \frac{d r}{d \xi}\right)+\frac{1}{4} \Phi^{\prime \prime} r-\gamma^{2} S_{\gamma}^{(1)}=0,
$$

где

$$
S_{\gamma}^{(1)}=-\frac{1}{4} \Phi\left(\Phi^{\prime} r^{\prime}+\frac{1}{2} \Phi^{\prime \prime} r\right) .
$$

Величина $\gamma^{2} S_{\gamma}^{(1)}$, как видно из $(20)-(22)$, определяет величину смещения гауссовой плоскости изображения в релятивистском случае относительно ее положения, определяемого уравнением параксиальных траекторий в нерелятивистском приближении:

$$
\sqrt{\Phi} \frac{d}{d \xi}\left(\sqrt{\Phi} \frac{d r}{d \zeta}\right)+\frac{1}{4} \Phi^{\prime \prime} r=0
$$

С учетом (20), (21) уравнение (13) принимает вид

$$
\sqrt{\Phi} \frac{d}{d \xi}\left[\sqrt{\Phi} \frac{d r}{d \xi}\right]+\frac{1}{4} \Phi^{\prime \prime} r=S+\gamma^{2}\left(S_{\gamma}^{(1)}+S_{\gamma}^{(3)}\right) \text {. }
$$

Откуда с учетом очевидного неравенства $S_{\gamma}^{(3)} \ll S_{\gamma}^{(1)}$ получим

$$
\sqrt{\Phi} \frac{d}{d \xi}\left(\sqrt{\Phi} \frac{d r}{d \xi}\right)+\frac{1}{4} \Phi^{\prime \prime} r=S+\gamma^{2} S_{\gamma}^{(1)} .
$$

Следует отметить, что между уравнениями (13) и (25) имеется одно очень важное отличие. Уравнение (13) пригодно для интегрирования методом последовательных приближении при любых скоростях частиц, поскольку малость его правой части обеспечивается только условиями параксиального приближения, в то время как малость правой части уравнения (25) обеспечивается не только условиями параксиального приближения, но и требованием сравнительно невысоких скоростей поступающих в зеркало частиц: $\gamma^{2} \ll 1$ (случай субрелятивистского приближения).

В этом случае система уравнений (13) и (14) с учетом (20), (22) и (25) принимает вид

$$
\begin{gathered}
\Phi r^{\prime \prime}+\left(\frac{1}{2} \Phi^{\prime} r^{\prime}+\frac{1}{4} \Phi^{\prime \prime} r\right)\left(1+\frac{1}{2} \gamma^{2} \Phi\right) \\
=\frac{1}{32} \Phi^{I V} r^{3}-\frac{1}{4} \Phi^{\prime \prime \prime} r \eta, \\
2 \Phi \eta^{\prime}-\Phi^{\prime} \eta=\varepsilon-\frac{1}{4} \Phi^{\prime \prime} r^{2}-\Phi r^{\prime 2} .
\end{gathered}
$$

\section{3. Интегрирование уравнений траекторий}

Уравнения (26), (27) записаны в такой форме, которая при указанных условиях допускает их решение методом последовательных приближений. При этом малость правых частей этих уравнений обеспечивается малостью $\mathrm{dfвеличины} \sqrt{\Phi} r^{\prime}$. Малость величины $\sqrt{\Phi} r^{\prime}$ обусловлена либо малостью $\Phi$ в окрестности особой точки $\xi=z_{u}$, где $\Phi\left(z_{u}\right)=0$, а $r^{\prime}$ в общем случае не может считаться малой, либо малостью $r^{\prime}$ в удаленных от особой точки областях.

Для решения системы уравнений (26), (27) сначала найдем величину $U=U(\xi)$, определяющую $r$ в первом приближении, положив правую часть (26) равной нулю. Подставив $U$ в правую часть уравнения (27), вычислим $\eta=\eta(\xi)$. Далее, подставив $U$ и $\eta$ в правую часть уравнения (26), найдем частное решение $\chi-\chi(\xi)$ уравнения (26). При этом общее решение этого уравнения будет иметь вид

$$
r=U+\chi
$$


При равенстве нулю правой части (26) имеем линейное однородное дифференциальное уравнение первого порядка

$$
\bar{\Phi} U^{\prime \prime}+\frac{1}{2} \Phi^{\prime} U^{\prime}+\frac{1}{4} \Phi^{\prime \prime} U=0,
$$

где

$$
\bar{\Phi}=\Phi /\left(1+\frac{1}{2} \gamma^{2} \Phi\right) .
$$

В случае электронного зеркала, как указано выше, на главной оптической оси системы имеется точка $\xi=z_{u}$, в которой $\Phi\left(z_{u}\right)=0$, а $\Phi^{\prime}\left(z_{u}\right) \neq 0$. Эта точка является регулярной особой точкой для коэффициентов уравнения (29) при $U$ и $U^{\prime}$. В соответствии с теорией такого рода уравнений [13] выберем два линейно независимых частных решения этого уравнения, из которых одно $p=p(\xi)$ является аналитической функцией, а другое может быть представлено в виде

$$
g=q \sqrt{\Phi},
$$

где $q=q(\xi)$ - также аналитическая функция, удовлетворяющая уравнению

$$
\bar{\Phi} q^{\prime \prime}+\frac{3}{2} \Phi^{\prime} q^{\prime}+\frac{3}{4} \Phi^{\prime \prime} q=0,
$$

следующему из (29) и (31).

Зададим начальные условия в особой точке $\xi=z_{u}$ :

$$
p_{u}=q_{u}=1 .
$$

В точке $\xi=z_{u}$ аналитические функции $p=p(\xi)$ и $q=q(\zeta)$ имеют все производные, первая из которых имеет следующий вид:

$$
p_{u}^{\prime}=q_{u}^{\prime}=-\frac{1}{2} \frac{\Phi_{u}^{\prime \prime}}{\Phi_{u}^{\prime}} .
$$

Здесь и далее индексом „, $u^{“}$ отмечены значения величин в точке $\xi=z_{u}$.

Используя эти частные решения как фундаментальную систему решений уравнения (29), общее решение этого уравнения представим в виде

$$
U=a p+b g,
$$

где $a$ и $b-$ произвольные постоянные.

С учетом (35) решение уравнения (27) представим в виде

$$
\eta=a^{2} \eta_{p}+a b \eta_{p g}+b^{2} \eta_{g}+\varepsilon \eta_{\varepsilon},
$$

где $\eta_{p}, \eta_{p g}, \eta_{g}, \eta_{\varepsilon}$ - решения линейных неоднородных дифференциальных уравнений первого порядка

$$
2 \Phi \eta_{j}^{\prime}-\Phi^{\prime} \eta_{J}=-F_{j}, \quad(j=p, p g, g, \varepsilon) .
$$

Здесь и далее

$$
F_{p}=\frac{1}{4} \Phi^{\prime \prime} p^{2}+\Phi p^{\prime 2}, \quad \frac{1}{2} F_{p g}=\frac{1}{4} \Phi^{\prime \prime} p g+\Phi p^{\prime} g^{\prime},
$$

$$
F_{g}=\frac{1}{4} \Phi^{\prime \prime} g^{2}+\Phi g^{2}, \quad F_{\varepsilon}=-1 .
$$

При этом в точке $\xi=z_{u}$ имеет место равенство

$$
\eta_{j u}=\frac{F_{j u}}{\Phi_{u}^{\prime}} \quad(j=p, p g, g, \varepsilon) .
$$

В особой точке $\xi=z_{u}$ коэффициенты уравнений (37) при $\eta_{p}, \eta_{p g}, \eta_{g}, \eta_{\varepsilon}$ и в свободном члене обращаются в бесконечность. Поэтому для упрощения решения уравнения (37) перепишем его путем умножения на интегрирующий множитель $\frac{1}{2 \Phi \sqrt{\Phi}}$ в виде уравнений в полных дифференциалах:

$$
\begin{gathered}
\left(\frac{\eta_{j}-\eta_{j u}}{\sqrt{\Phi}}\right)^{\prime}=-\frac{1}{2 \Phi \sqrt{\Phi}}\left(F_{j}-F_{j u} \frac{\Phi^{\prime}}{\Phi_{u}^{\prime}}\right), \\
(j=p, p g, g, \varepsilon) .
\end{gathered}
$$

Решая эти уравнения с учетом (29) и (32), получим

$$
\begin{gathered}
\eta_{p}=-\frac{1}{2} p p^{\prime}+\frac{\sqrt{\Phi}}{2} \int_{z_{u}}^{\xi} \frac{p p^{\prime \prime}}{\sqrt{\Phi}}\left(1+\frac{\bar{\Phi}}{\Phi}\right) d \xi \\
\eta_{p g}=\sqrt{\Phi} p_{u}^{\prime}-g p^{\prime}+\sqrt{\Phi} \int_{z_{u}}^{\xi} q p^{\prime \prime}\left(1+\frac{\bar{\Phi}}{\Phi}\right) d \xi \\
n_{g}=\frac{1}{4} \frac{\bar{\Phi}}{\Phi} \Phi^{\prime} q^{2}-\frac{1}{2} \Phi q q^{\prime}+\frac{\sqrt{\Phi}}{2} \int_{z_{u}}^{\xi} \sqrt{\Phi} q q^{\prime \prime}\left(1+\frac{\bar{\Phi}}{\Phi}\right) d \xi \\
n_{\varepsilon}=-\frac{1}{\Phi_{u}^{\prime}}\left[1+\frac{\sqrt{\Phi}}{2} \int_{z_{u}}^{\xi} \frac{1}{\Phi \sqrt{\Phi}}\left(\Phi^{\prime}-\Phi_{u}^{\prime}\right) d \xi\right]
\end{gathered}
$$

Отметим, что величины $\eta_{p}, \eta_{p g}, \eta_{g}, \eta_{\varepsilon}$ повсюду конечны вместе со своими производными.

Теперь определим частное решение $\chi=\chi(\xi)$ уравнения (26). С учетом (35) и (36) представим его в виде

$$
\begin{aligned}
\chi= & a^{3} \chi_{1}+a^{2} b\left(\chi_{2}+\chi_{3}\right)+a b^{2}\left(\chi_{4}+\chi_{5}\right) \\
& +b^{3} \chi_{6}+\varepsilon\left(a \chi_{7}+b \chi_{8}\right),
\end{aligned}
$$

где $\chi_{m}=\chi_{m}(\xi)(m=1-8)-$ решения линейных неоднородных дифференциальных уравнений второго порядка

$$
\begin{gathered}
\Phi \chi_{m}^{\prime \prime}+\left(\frac{1}{2} \Phi^{\prime} \chi_{m}^{\prime}+\frac{1}{4} \Phi^{\prime \prime} \chi_{m}\right)\left(1+\frac{1}{2} \gamma^{2} \Phi\right)=S_{m} \\
(m=1-8) .
\end{gathered}
$$

Здесь

$$
\begin{array}{cl}
S_{1}=p S_{p}, & S_{2}=g S_{p}, \\
S_{3}=p S_{p g}, & S_{4}=g S_{p g}, \\
S_{5}=p S_{g}, & S_{6}=g S_{g},
\end{array}
$$




$$
S_{7}=p S_{\varepsilon}, \quad S_{8}=g S_{\varepsilon},
$$

где

$$
\begin{gathered}
S_{p}=\frac{1}{32} \Phi^{I V} p^{2}-\frac{1}{4} \Phi^{\prime \prime \prime} \eta_{p}, \\
S_{p g}=\frac{1}{16} \Phi^{I V} p g-\frac{1}{4} \Phi^{\prime \prime \prime} \eta_{p g}, \\
S_{g}=\frac{1}{32} \Phi^{I V} g^{2}-\frac{1}{4} \Phi^{\prime \prime \prime} \eta_{g}, \\
S_{\varepsilon}=\frac{1}{32} \Phi^{\prime \prime \prime} \eta_{\varepsilon} .
\end{gathered}
$$

Решая уравнения (46) методом вариации произвольных постоянных, получим

$$
\chi_{j}=-\frac{1}{W}\left[p \int_{z_{u}}^{\xi} \frac{g S_{j}}{\sqrt{\Phi}} d \xi-g \int_{z_{u}}^{\xi} \frac{p S_{j}}{\sqrt{\Phi}} d \xi\right]
$$

где $W$ - инвариант Вронского:

$$
W=\sqrt{\Phi}\left(p g^{\prime}-p^{\prime} g\right)=\frac{1}{2} \Phi_{u}^{\prime} .
$$

Подынтегральные выражения в (49), как видно из (47), (48), содержат величины $\eta_{p}, \eta_{p g}, \eta_{g}, \eta_{\varepsilon}$, которые, в свою очередь, определены через интегралы (41)-(44). Численный расчет двойных интегралов связан с определенными трудностями. Для приведения частных решений $\chi_{m}=\chi_{m}(\xi)(m=1-8)$ к удобному для численных расчетов виду выполнены следующие преобразования. Во-первых, от таких двойных интегралов можно освободиться интегрированием по частям с помощью равенств, следующих из (37), (38):

$$
\begin{aligned}
& \frac{1}{4} \Phi^{\prime \prime \prime} p^{2}=F_{p}^{\prime}, \quad \frac{1}{4} \Phi^{\prime \prime \prime} p g=\frac{1}{2} F_{p g}^{\prime}, \quad \frac{1}{4} \Phi^{\prime \prime \prime} g^{2}=F_{g}^{\prime}, \\
& F_{l}^{\prime}\left(\frac{\eta_{\varepsilon}}{\sqrt{\Phi}}\right)=\left(\frac{F_{l} \eta_{\varepsilon}+\eta_{l}}{\sqrt{\Phi}}\right)^{\prime}, \quad(l=p, p g, g) .
\end{aligned}
$$

Кроме того, используя равенства

$$
\begin{aligned}
p S_{2}=g S_{1}, \quad p S_{4}=g S_{3}, \quad p S_{6}=g S_{5}, \quad p S_{8}=g S_{7} \\
g S_{4}-2 p S_{6}=\frac{1}{4} \Phi^{\prime \prime \prime}\left(2 p g \eta_{g}-g^{2} \eta_{p g}\right) \\
=2 \sqrt{\Phi}\left[\sqrt{\Phi}\left(\eta_{p g} \eta_{g}^{\prime}-\eta_{g} \eta_{p g}^{\prime}\right)\right]^{\prime} \\
p S_{3}-2 g S_{1}=\frac{1}{4} \Phi^{\prime \prime \prime}\left(2 p g \eta_{g}-p^{2} \eta_{p g}\right) \\
=2 \sqrt{\Phi}\left[\sqrt{\Phi}\left(\eta_{p g} \eta_{p}^{\prime}-\eta_{p} \eta_{p g}^{\prime}\right)\right]^{\prime} \\
g S_{2}-2 p S_{5}=\frac{1}{4} \Phi^{\prime \prime \prime}\left(p^{2} \eta_{g}-g^{2} \eta_{p}\right) \\
=2 \sqrt{\Phi}\left[\sqrt{\Phi}\left(\eta_{p} \eta_{g}^{\prime}-\eta_{g} \eta_{p}^{\prime}\right)\right]^{\prime}
\end{aligned}
$$

следующие также из равенств (37), (38) и (41)-(44), можно сократить количество интегралов, определяющих $\chi_{m}=\chi_{m}(\zeta)(m=1-8)$.

Далее, используя очевидные равенства

$$
\begin{aligned}
& \frac{1}{2} p F_{p g}-g F_{p}=\sqrt{\Phi} W p^{\prime}, \\
& p F_{g}-\frac{1}{2} g F_{p g}=\sqrt{\Phi} W g^{\prime},
\end{aligned}
$$

следующие из (29) и (38), решения $\chi_{m}=\chi_{m}(\xi)$ $(m=1-8)$ можно записать в виде

$$
\begin{gathered}
\chi_{1}=\eta_{p} p^{\prime}+p J_{2}-g J_{1}, \\
\chi_{2}=\eta_{p} g^{\prime}+p J_{4}-g\left(J_{2}-\frac{1}{2} p^{2}\right), \\
\chi_{3}=\eta_{p g} p^{\prime}+2\left[p J_{3}-g\left(J_{2}-\frac{1}{2} p^{\prime 2}\right)\right], \\
\chi_{4}=\eta_{p g} g^{\prime}+2\left[p J_{5}-g\left(J_{3}-\frac{1}{2} p^{\prime} g^{\prime}\right)\right], \\
\chi_{5}=\eta_{g} p^{\prime}+p J_{5}-g\left(J_{4}-\frac{1}{2} p^{\prime} g^{\prime}\right), \\
\chi_{6}=\eta_{g} g^{\prime}+p J_{6}-g\left(J_{5}-\frac{1}{2} g^{\prime 2}\right), \\
\chi_{7}=\eta_{\varepsilon} p^{\prime}+p J_{8}-g J_{7}, \\
\chi_{8}=\eta_{\varepsilon} g^{\prime}+p J_{9}-g\left(J_{8}-\frac{1}{2 \Phi}\right) .
\end{gathered}
$$

Здесь

$$
\begin{gathered}
J_{1}=-\frac{1}{\Phi_{u}^{\prime}} \int_{z_{u}}^{\infty} \frac{1}{\sqrt{\Phi}}\left[L_{p} p^{3}+\left(1+\frac{\bar{\Phi}}{\Phi}\right) F_{p} p p^{\prime \prime}\right] d \xi \\
J_{2}=-\frac{1}{\Phi_{u}^{\prime}} \int_{z_{u}}^{\infty} \frac{1}{\Phi}\left[L_{p} p^{2} g+\left(1+\frac{\bar{\Phi}}{\Phi}\right) F_{p} g p^{\prime \prime}\right] d \xi \\
+\frac{1}{4}\left(1-\frac{\bar{\Phi}}{\Phi}\right) p^{\prime 2}, \\
J_{3}=-\frac{1}{\Phi_{u}^{\prime}} \int_{z_{u}}^{\infty} \frac{1}{\Phi}\left[L_{p} p g^{2}+\frac{1}{2}\left(1+\frac{\bar{\Phi}}{\Phi}\right) F_{p g} g p^{\prime \prime}\right] d \zeta, \\
J_{4}=-\frac{1}{\Phi_{u}^{\prime}} \int_{z_{u}}^{\infty}\left[L_{g} p^{2} g+\left(1+\frac{\bar{\Phi}}{\Phi}\right) F_{p} g q^{\prime \prime}\right] d \zeta, \\
J_{5}=-\frac{1}{\Phi_{u}^{\prime}} \int_{z_{u}}^{\infty}\left[L_{g} p g^{2}+\frac{1}{2}\left(1+\frac{\bar{\Phi}}{\Phi}\right) F_{p g} g q^{\prime \prime}\right] d \xi, \\
J_{6}=-\frac{1}{\Phi_{u}^{\prime}} \int_{z_{u}}^{\infty}\left[L_{g} g^{3}+\left(1+\frac{\bar{\Phi}}{\Phi}\right) F_{g} g q^{\prime \prime}\right] d \xi,
\end{gathered}
$$




$$
\begin{gathered}
J_{7}=\frac{1}{\Phi_{u}^{\prime}} \int_{z_{u}}^{\infty} \frac{p p^{\prime \prime}}{\sqrt{\Phi}}\left(1+\frac{\bar{\Phi}}{\Phi}\right) d \xi, \\
J_{8}=\frac{1}{\Phi_{u}^{\prime}} \int_{z_{u}}^{\infty} q p^{\prime \prime}\left(1+\frac{\bar{\Phi}}{\Phi}\right) d \xi, \\
J_{9}=\frac{1}{\Phi_{u}^{\prime}} \int_{z_{u}}^{\infty} \sqrt{\Phi} q q^{\prime \prime}\left(1+\frac{\bar{\Phi}}{\Phi}\right) d \xi,
\end{gathered}
$$

где

$$
\begin{gathered}
L_{p}=\frac{1}{16}\left(\Phi^{I V} p+4 \Phi^{\prime \prime \prime} p^{\prime}\right), \\
L_{g}=\frac{1}{16}\left[\left(\Phi^{I V}-2 \frac{\bar{\Phi}}{\Phi} \frac{\Phi^{\prime \prime \prime} \Phi^{\prime}}{\Phi}\right) q+4 \Phi^{\prime \prime \prime} q^{\prime}\right] .
\end{gathered}
$$

Здесь и далее знак „ $\infty$ означает, что верхняя граница интегрирования отодвинута на бесконечность. Это, очевидно, не может изменить результата, так как подынтегральная функция равна нулю в свободных от поля областях.

С учетом (36) и (57)-(64), можно записать (45) в виде

$$
\chi=\eta\left(a p^{\prime}+b g^{\prime}\right)+\Lambda,
$$

где $\Lambda=\Lambda(\xi)$ - величина, не содержащая динамическую переменную $\eta=\eta(\xi)$ :

$$
\Lambda=a^{3} \Lambda_{1}+a^{2} b \lambda_{2}+a b^{2} \Lambda_{3}+b^{3} \Lambda_{4}+\varepsilon\left(a \Lambda_{5}+b \Lambda_{6}\right) .
$$

Здесь

$$
\begin{gathered}
\Lambda_{1}=p J_{2}-g J_{1}, \\
\Lambda_{2}=p\left(2 J_{3}+J_{4}\right)-3 g\left(J_{2}-\frac{1}{2} p^{\prime 2}\right), \\
\Lambda_{3}=3 p J_{5}-g\left(2 J_{3}+J_{4}-\frac{3}{2} g^{\prime} p^{\prime}\right), \\
\Lambda_{4}=p J_{6}-g\left(J_{5}-\frac{1}{2} p^{2}\right) \\
\Lambda_{5}=p J_{8}-g J_{7}, \\
\Lambda_{6}=p J_{9}-g\left(J_{8}+\frac{1}{2 \Phi}\right)
\end{gathered}
$$

Таким образом, в подвижной системе координат уравнения траекторий частиц в поле зеркала от особой точки $\xi=z_{u}$ до произвольной точки $\xi=$ const описываются системой уравнений

$$
\begin{aligned}
& r=a p(\xi)+b g(\xi)+\eta(\xi)\left[a p^{\prime}(\xi)+b g^{\prime}(\xi)\right]+\Lambda, \\
& z=\xi+a^{2} \eta_{p}(\xi)+a b \eta_{p g}(\xi)+b^{2} \eta_{g}(\xi)+\varepsilon \eta_{\varepsilon}(\xi),
\end{aligned}
$$

следующих из равенств (6), (28), (35), (36) и (75).

\section{4. Уравнения траекторий в лабораторной системе координат}

Система уравнений (83), (84) определяет в параметрическом виде траектории частиц в движущейся системе координат в зависимости от положения $\xi$ на главной оптической оси $z$ некоторой выбранной (центральной) частицы. Для того чтобы получить уравнение траекторий частиц $r=r(z)$ в виде явной зависимости от координаты $z$ оптической оси системы, выполним следующие преобразования. Разрешим уравнение (84) относительно $\xi$ и подставим зависимость $\xi=z-\eta(z)$ в уравнение (83). Затем удержим в разложении величины не выше третьего порядка малости относительно начальных параметров движения частицы $a, b, \varepsilon$.

После выполнения этих преобразований уравнения траекторий принимает обычный для лабораторной системы координат вид

$$
r=a p+b g+\Lambda .
$$

Здесь и далее $p=p(z), g=g(z), \Lambda=\Lambda(z)-$ функции, ранее определенные как функции от $\xi$.

Для определения конкретной траектории необходимо выразить произвольные постоянные $a$ и $b$ через начальные условия. При этом необходимо учесть, что электронные зеркала характеризуются наличием двух ветвей траектории: прямой - от начальной (предметной) плоскости до точки поворота, и обратной - от точки поворота до произвольной плоскости. При этом, как следует из условия непрерывности траектории частицы и ее скорости в окрестности точки поворота, различие уравнений для прямой и обратной ветвей траектории состоит лишь в том, что значения постоянной $a$ для прямой и обратной ветвей траектории совпадают, а значения постоянной $b-$ различаются знаком [10]. Тогда уравнения траектории и ее наклона к оси $z$ c точностью до величин третьего порядка малости можно записать в виде

$$
\begin{gathered}
r=a p(z) \pm b g(z)+\Lambda, \\
r^{\prime}=a p^{\prime}(z) \pm b g^{\prime}(z)+\Lambda^{\prime},
\end{gathered}
$$

где

$$
\begin{aligned}
& \Lambda=a^{3} \Lambda_{1} \pm a^{2} b \Lambda_{2}+a b^{2} \Lambda_{3} \pm b^{3} \Lambda_{4}+\varepsilon\left(a \Lambda_{5} \pm b \Lambda_{6}\right) \\
& \Lambda^{\prime}=a^{3} \Lambda_{1}^{\prime} \pm a^{2} b \Lambda_{2}^{\prime}+a b^{2} \Lambda_{3}^{\prime} \pm b^{3} \Lambda_{4}^{\prime}+\varepsilon\left(a \Lambda_{5}^{\prime} \pm b \Lambda_{6}^{\prime}\right) .
\end{aligned}
$$

Здесь и далее при двойном знаке „士“ \pm знак „+“ относится к прямой ветви траектории, а знак „-“ обратной, а штрихи обозначают дифференцирование по переменной $z$.

Решая систему уравнений (86), (87) для прямой ветви, получим

$$
a=\alpha+\frac{2}{\Phi_{u}^{\prime}}\left(g_{0} \Lambda_{0}^{\prime}-g_{0}^{\prime} \Lambda_{0}\right),
$$




$$
b=\beta-\frac{2}{\Phi_{u}^{\prime}}\left(p_{0} \Lambda_{0}^{\prime}-p_{0}^{\prime} \Lambda_{0}\right)
$$

где

$$
\alpha=-\frac{2}{\Phi_{u}^{\prime}}\left(g_{0} r_{0}^{\prime}-g_{0}^{\prime} r_{0}\right), \quad \beta=\frac{2}{\Phi_{u}^{\prime}}\left(p_{0} r_{0}^{\prime}-p_{0}^{\prime} r_{0}\right) .
$$

Здесь и далее индексом „,“ отмечены значения величин в начальной (предметной) плоскости $z=z_{0}$.

С учетом (90), (91), уравнение траектории для обратной ветви можно записать в виде

$$
r=U+\Delta r
$$

где

$$
U=-\frac{2}{\Phi_{u}^{\prime}}\left[r_{0}^{\prime}\left(p_{0} g+p g_{0}\right)-r_{0}\left(p_{0}^{\prime} g+p g_{0}^{\prime}\right)\right]
$$

— уравнение параксиальной траектории,

$$
\begin{aligned}
& \Delta r=\Lambda+\frac{2}{\Phi_{u}^{\prime}} \\
& \times\left[\Lambda_{0}^{\prime}\left(p_{0} g+p g_{0}\right)-\Lambda_{0}\left(p_{0}^{\prime} g+p g_{0}^{\prime}\right)\right]
\end{aligned}
$$

- суммарная пространственная аберрация.

\section{2. Электронно-оптические свойства зеркала}

\section{1. Пространственная фокусировка}

Условие пространственной фокусировки частиц, как это видно из (93), определяется равенством

$$
p_{0} g\left(z_{G}\right)+p\left(z_{G}\right) g_{0}=0,
$$

где $z=z_{G}-$ положение гауссовой плоскости изображения зеркала.

Откуда с учетом (93) следует

$$
\frac{p\left(z_{G}\right)}{p_{0}}=-\frac{g\left(z_{G}\right)}{g_{0}}=\frac{U}{r_{0}}=M
$$

где $M$ - линейное увеличение зеркала.

Как видно из (93), условие фокусировки параллельных пучков частиц определяется равенством

$$
p_{0}^{\prime} g\left(z_{F}\right)+p\left(z_{F}\right) g_{0}^{\prime}=0,
$$

где $z=z_{F}$ - положение фокальной плоскости зеркала.

Откуда с учетом (93) следует

$$
\frac{p\left(z_{F}\right)}{p_{0}^{\prime}}=-\frac{g\left(z_{F}\right)}{g_{0}^{\prime}}=\frac{U}{r_{0}^{\prime}}=f,
$$

где $f$ - фокусное расстояние зеркала.

\section{2. Кардинальные элементы зеркала}

Для определения кардинальных элементов воспользуемся характерными для зеркала траекториями. Решение $p=p(z)$ описывает траектории, прямые и обратные ветви которых совпадают, т.е. проходят через центр кривизны зеркала $z=z_{C}$, а решение $g=g(z)$ - траектории, прямые и обратные ветви которых симметричны относительно оптической оси зеркала, т.е. проходят через вершину зеркала $z=z_{V}$ [10]. Таким образом, когда предмет и его изображение находятся в свободном от поля пространстве, функции $p=p(z)$ и $g=g(z)$ можно записать в виде

$$
p=\left(z-z_{C}\right) p^{\prime}, \quad g=\left(z-z_{V}\right) g^{\prime} .
$$

С учетом этих равенств из (97), (98) следует, что положение фокуса зеркала и его фокусное расстояние определяются равенствами

$$
z_{F}=\frac{1}{2}\left(z_{V}+z_{C}\right), \quad f=\frac{1}{2}\left(z_{V}-z_{C}\right) .
$$

\section{3. Аберрации}

Суммарная аберрация (94) в гауссовой плоскости с учетом (95), (96) принимает вид

$$
\Delta r=\Lambda-M \Lambda_{0}
$$

Перепишем это равенство с учетом (88) в виде

$$
\Delta r=\alpha^{3} G_{1}+\alpha^{2} \beta G_{2}+\alpha \beta^{2} G_{3}+\beta^{3} G_{4}+\varepsilon\left(\alpha G_{5}+\beta G_{6}\right),
$$

где

$$
\begin{array}{r}
G_{i}=\Lambda_{i}-M \Lambda_{0 i}, \quad(i=1,3,5), \\
G_{i}=-\left(\Lambda_{i}+M \Lambda_{0 i}\right), \quad(i=2,4,6) .
\end{array}
$$

Откуда с учетом (77)-(82) и (96) получим

$$
\begin{gathered}
G_{1}=2 M g_{0} J_{1}, \quad G_{2}=-2 M p_{0}\left(2 J_{3}+J_{4}\right), \\
G_{3}=2 M g_{0}\left(2 J_{3}+J_{4}-\frac{3}{2} g^{\prime} p^{\prime}\right), \quad G_{4}=-2 M p_{0} J_{6}, \\
G_{5}=2 M g_{0} J_{7}, \quad G_{6}=-2 M p_{0} J_{9} .
\end{gathered}
$$

После громоздких, но несложных преобразований с учетом (91) получим

$$
\begin{aligned}
\Delta r & =M\left[r_{0}^{\prime 3} B+3 r_{0}^{\prime 2} r_{0} F+r_{0}^{\prime} r_{0}^{2}(2 C+D)\right. \\
& \left.+r_{0}^{3} E+\varepsilon\left(r_{0}^{\prime} K_{1}+r_{0} K_{2}\right)\right]
\end{aligned}
$$

где $B, F, C, D, E$ - постоянные геометрических аберраций зеркала: сферической, комы, астигматизма, кривизны поля изображения, дисторсии, а $K_{1}$ и $K_{2}-$ постоянные хроматической аберрации двух видов (осевой и изменения увеличений):

$$
B=\frac{1}{R^{3}}\left[Z_{V}^{4} \bar{J}_{1}+2 Z_{V}^{2} Z_{C}^{2}\left(2 \bar{J}_{3}+\bar{J}_{4}\right)+Z_{C}^{4} \bar{J}_{6}+3 Z_{V}^{2} Z_{C}^{2}\right],
$$




$$
\begin{gathered}
F=-\frac{1}{R^{3}}\left[Z_{V}^{2} \bar{J}_{1}+2\left(2 \bar{J}_{3}+\bar{J}_{4}\right) Z_{V} Z_{C} Z_{F}\right. \\
\left.+Z_{C}^{3} \bar{J}_{6}+Z_{V} Z_{C}\left(2 Z_{V}+Z_{C}\right)\right] \\
C=\frac{1}{R^{3}}\left[Z_{V}^{2} \bar{J}_{1}+2 Z_{V} Z_{C}\left(2 \bar{J}_{3}+\bar{J}_{4}\right)\right. \\
\left.+Z_{C}^{2} \bar{J}_{6}+Z_{V}\left(2 Z_{C}+Z_{V}\right)\right] \\
D=\frac{1}{R^{3}}\left[Z_{V}^{2} \bar{J}_{1}+\left(Z_{V}^{2}+Z_{C}^{2}\right)\left(2 \bar{J}_{3}+\bar{J}_{4}\right)\right. \\
\left.+Z_{C}^{2} \bar{J}_{6}+Z_{V}\left(2 Z_{C}+Z_{V}\right)\right] \\
\left.E=\frac{1}{R^{3}}\left[Z_{V} \bar{J}_{1}+2 Z_{F}\left(2 \bar{J}_{3}+\bar{J}_{4}\right)+Z_{C} \bar{J}_{6}+3 Z_{V}\right)\right], \\
K_{1}=\frac{1}{R}\left(Z_{V}^{2} \bar{J}_{7}+Z_{C}^{2} \bar{J}_{9}\right) \\
K_{2}=-\frac{1}{R}\left(Z_{V} \bar{J}_{7}+Z_{C} \bar{J}_{9}\right) .
\end{gathered}
$$

где $R=z_{V}-z_{C}-$ радиус зеркала, $Z_{V}=z_{0}-z_{V}$, $Z_{C}=z_{0}-z_{c}, Z_{F}=z_{0}-z_{F}, \mathrm{a}$

$$
\begin{gathered}
\bar{J}_{1}=-\frac{2 g_{0}^{\prime}}{p_{0}^{\prime 3}} J_{1}, \quad \bar{J}_{3}=-\frac{2}{p_{0}^{\prime} g_{0}^{\prime}} J_{3}, \quad \bar{J}_{4}=-\frac{2}{p_{0}^{\prime} g_{0}^{\prime}} J_{4}, \\
\bar{J}_{6}=-\frac{2 p_{0}^{\prime}}{g_{0}^{\prime 3}} J_{6}, \quad \bar{J}_{7}=-\frac{2 g_{0}^{\prime}}{p_{0}^{\prime}} J_{7}, \quad \bar{J}_{9}=-\frac{2 p_{0}^{\prime}}{g_{0}^{\prime}} J_{9} .
\end{gathered}
$$

Заметим, что выражения для постоянных аберраций (106)-(112) в нерелятивистском приближении полностью совпадают с соответствующими выражениями [10] для электростатического зеркала.

\section{3. Трехэлектродное зеркало, свободное от сферической и осевой хроматической аберраций при учете релятивизма}

В настоящей работе рассчитано трехэлектродное зеркало вращательной симметрии, состоящее из соосных цилиндров равного диаметра $d$, находящихся под потенциалами $V_{1}, V_{2}, V_{3}$ (рис. 1). При этом рассмотрен режим работы зеркала, когда начальная (предметная) плоскость

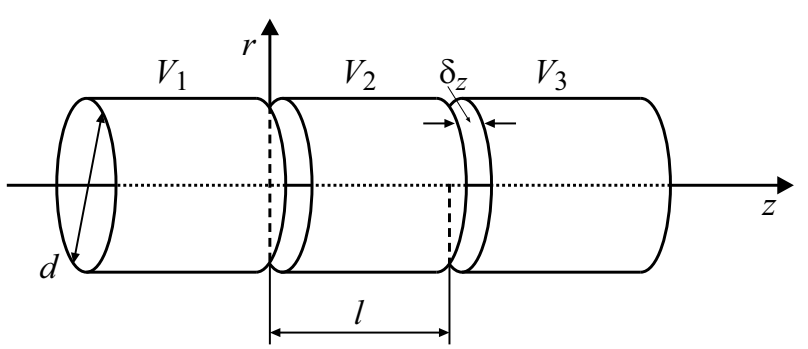

Рис. 1. Трехэлектродное зеркало вращательной симметрии. $V_{1}, V_{2}, V_{3}$ - потенциалы на электродах, $d$ - диаметр цилиндра, $l$ - длина среднего электрода, $\delta_{z}$ - ширина зазора между электродами. совмещена с фокальной плоскостью зеркала, как и в работе [14].

Осевое распределение электростатического потенциала такого зеркала описывается соотношением [15]:

$$
\Phi(z)=\frac{1}{2}\left[\left(V_{1}+V_{3}\right)+\sum_{j=1}^{2}\left(V_{i+1}-V_{i}\right) U\left(z-z_{i}\right)\right],
$$

где

$$
\begin{gathered}
U\left(z-z_{i}\right)=\operatorname{sign}\left(z-z_{i}\right) \\
\sum_{s=1}^{\infty}\left[1-B_{s} \exp \left(-2 \alpha_{s}\left|z-z_{i}\right| / d\right)\right], \\
B_{s}=\prod_{m=1}^{\infty}\left(1-\alpha_{s}^{2} / \alpha_{m}^{2}\right)_{s \neq m}^{-1} .
\end{gathered}
$$

Здесь $z_{i}$ - координата середины $i$-го зазора между электродами, $\alpha_{s}, \alpha_{m}$ - корни функции Бесселя нулевого порядка. Принято, что положительное направление оси $z$ совпадает с направлением движения падающих на зеркало ионов, а начало координат помещено в середине первого зазора (между первым и вторым электродами).

Расчет зеркала производился следующим образом. Для значения ширины среднего электрода $l=0.6 d$ находились значения положения предметной плоскости, совмещенной с фокальной плоскостью зеркала, а также значения потенциала $V_{2}$ на втором (среднем) электроде и запирающего потенциала $V_{3}$ на третьем электроде, обеспечивающие выполнение условий устранения сферической и осевой хроматической аберрации одновременно в зависимости от значения безразмерной величины $\gamma^{2}$, представляющей собой количественную характеристику релятивистских эффектов поступающих в поле зеркала частиц.

Результаты расчета представлены на рис. 2-4. При этом на рис. 2 и 3 приведены значения положения

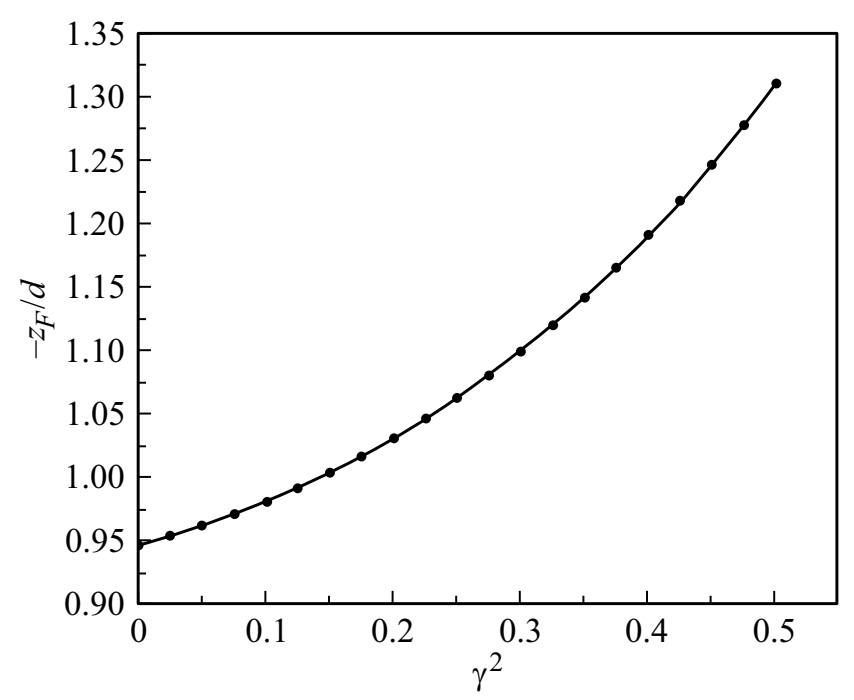

Рис. 2. Зависимость положения фокуса зеркала $z_{F}$ от величины $\gamma^{2}$. 


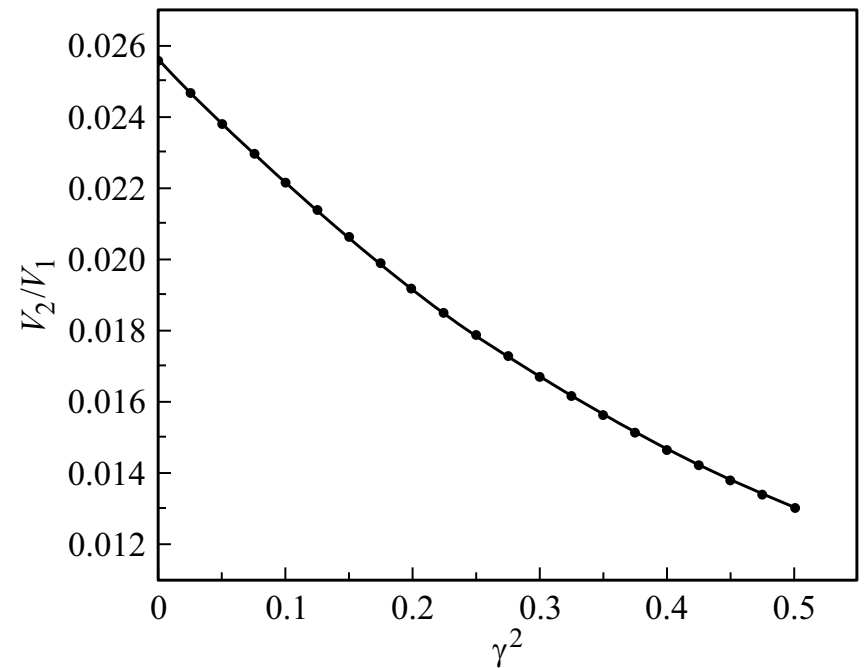

Рис. 3. Зависимость потенциала на среднем электроде $V_{2}$ от величины $\gamma^{2}$.

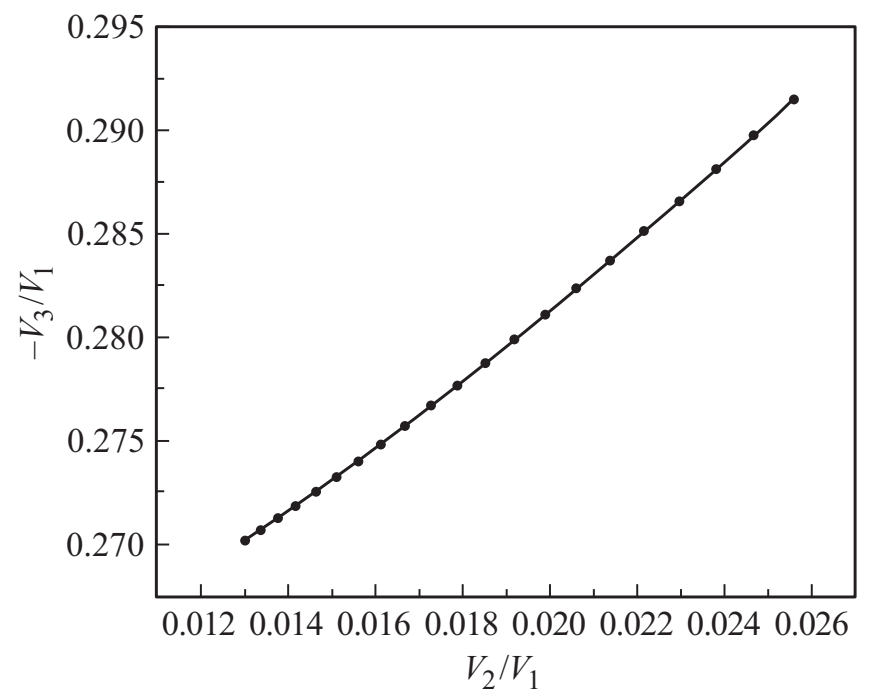

Рис. 4. Зависимость запирающего потенциала $V_{3}$ от потенциала $V_{2}$.

фокуса зеркала $z_{F}$ и потенциала на среднем электроде в зависимости от безразмерной величины $\gamma^{2}$, а на рис. $4-$ зависимость запирающего потенциала $V_{3}$ на третьем электроде от потенциала $V_{2}$.

Заметим, что в нерелятивистском приближении результаты данных расчетов полностью совпадают с результатами [14].

\section{Заключение}

Известный метод теоретического исследования фокусировки пучков заряженных частиц, опирающийся на представление динамических уравнений в движущейся системе координат, связанной с „центральной“ частицей, обобщен на случай суб-релятивистских скоростей ча- стиц. Такое представление существенно расширяет возможности теории фокусировки и позволяет выполнить аберрационный анализ электронно-оптической системы в экстремальных случаях, когда кинетическая энергия в процессе движения частиц изменяется в предельно широком диапазоне энергий - от нулевых значений до величин, сравнимых с энергией покоя электрона. Такие ситуации возникают, например, в эмиссионных системах при ускорении пучков до больших энергий или при отражении релятивистских пучков в электростатических зеркалах.

Полученные результаты и формулы могут быть использованы при разработке новых схем корректоров сферохроматических аберраций электронных линз для нужд высоковольтной электронной микроскопии и пр. Необходимо отметить тот факт, что субрелятивистский режим работы электронного зеркала сопровождается не только появлением смещения положения гауссовой плоскости изображения, но также приводит к изменению электрических параметров зеркала, обеспечивающих условия одновременного устранения сферической и осевой хроматической аберраций. Учет этих изменений может привести к существенному улучшению качества электронного изображения за счет уменьшения основных сферохроматических электронно-оптических аберраций в сочетании с естественным уменьшением дифракционного рассеяния.

\section{Финансирование работы}

Работа выполнена при финансовой поддержке Комитета науки Министерства образования и науки Республики Казахстан (грант № АР05132483).

\section{Конфликт интересов}

Авторы заявляют, что у них нет конфликта интересов.

\section{Список литературы}

[1] П. Хокс, Э. Каспер. Основы электронной оптики (Мир, M., 1993)

[2] P.W. Hawkes. Phil. Trans. R. Soc. A, 367, 3637 (2009).

[3] M. Haider, H. Rose, S. Uhlemann, E. Schwan, B. Kabius, K. Urban. Ultramicroscopy, 75, 53 (1998). DOI:10.1016/S0304-3991(98)00048-54

[4] M. Haider, H. Muller, S. Uhlemann. Adv. Imaging Electron Phys., 153, 43 (2008).

[5] G.F. Rempfer, J. Appl. Phys., 67 (10), 6027 (1990).

[6] D. Preikszas, H. Rose. J. Electron Microsc., 46 (1), 1 (1997).

[7] P. Hartel, D. Preikszas, R. Spehr, H. Muller, H. Rose. Adv. Imaging Electron Phys., 120, 41 (2002).

[8] O. Krivanek, N. Dellby, R.J. Keyse, M. Murfitt, C. Own, Z. Szilagyi. Adv. Imaging Electron Phys., 153, 121 (2008).

[9] S.B. Bimurzaev, N.U. Aldiyarov, E.M. Yakushev. Microscopy, 66, 356 (2017).

[10] E.M. Yakushev, L.M. Sekunova. Adv. Electronics Electron Phys., 68 (5), 337 (1986). 
[11] E.M. Yakushev Adv. Imaging Electron Phys., 178, 147 (2013).

[12] В. Глазер. Основы электронной оптики (ГИТТЛ, М., 1957)

[13] Г. Корн, Т. Корн. Справочник по математике для научных работников и инженеров (Наука, М., 1968)

[14] S.B. Bimurzaev, G.S. Serikbaeva, E.M. Yakushev. J. Electron Microscopy, 52 (4), 365 (2003).

[15] Б.В. Бобыкин, Ю.А. Невинный, Е.М. Якушев. ЖТФ, 45, 2368 (1975). 